Instituto Internacional de Investigación y Desarrollo Tecnológico Educativo INDTEC, C.A.

DOI: https://doi.org/10.29394/Scientific.issn.2542-2987.2021.6.20.10.190-205

OAl-PMH: http://www.indteca.com/ojs/index.php/Revista Scientific/oai

Artículo Original / Original Article

\title{
Narrativa de madres sobre la enseñanza-aprendizaje remota de emergencia en docentes de nivel inicial
}

Autores: María Patricia Cucho Leyva Universidad Cesar Vallejo, UCV mariacuchol@ucvvirtual.edu.pe Lima, Perú https://orcid.org/0000-0002-4449-4909

Wilfredo Humberto Carcausto Calla Universidad César Vallejo, UCV wcarcaustocalla@ucvvirtual.edu.pe Lima, Perú https://orcid.org/0000-0002-3218-871X

\section{Resumen}

El objetivo del estudio fue describir a través de los relatos la percepción de madres de familias sobre la enseñanza-aprendizaje docente antes, durante y después de la pandemia. La metodología fue cualitativa, con un diseño narrativo. Participaron ocho madres de familias, de Lima, Perú en el año 2020, los datos fueron recogidos utilizando una guía de entrevista. En los resultados, del análisis de la percepción e instrucción del docente, surgieron tres categorías emergentes: enseñanza-aprendizaje docente antes, durante y después de la pandemia. Se concluyó que el conocimiento de las madres de familias sobre la enseñanza-aprendizaje docente antes de la pandemia, se realizaba en la escuela directamente con el docente, durante la pandemia perciben en los docentes que tienen mayor compromiso con la mejora del proceso enseñanza-aprendizaje y comprendieron que su apoyo es fundamental en este proceso, después de la pandemia observan que la enseñanza-aprendizaje debe darse en la escuela para una mejor interacción con su docente, manteniendo una comunicación permanente con las madres, con la finalidad de seguir apoyando a sus hijos en sus hogares.

Palabras clave: educación a distancia; enseñanza; aprendizaje; pandemia; participación de los padres.

Código de clasificación internacional: 5802.07 - Formación profesional.

Cómo citar este artículo:

Cucho, M., \& Carcausto, W. (2021). Narrativa de madres sobre la enseñanza-aprendizaje remota de emergencia en docentes de nivel inicial. Revista Scientific, 6(20), 190-205, e-ISSN: 2542-2987. Recuperado de: https://doi.org/10.29394/Scientific.issn.2542-2987.2021.6.20.10.190-205

Fecha de Recepción: 26-12-2020
Fecha de Aceptación: 18-03-2021
Fecha de Publicación: 05-05-2021 
OAl-PMH: http://www.indteca.com/ojs/index.php/Revista Scientific/oai

Artículo Original / Original Article

\title{
Mothers' narrative on emergency remote teaching-learning in initial level teachers
}

\begin{abstract}
The objective of the study was to describe through the stories the perception of mothers of families about the teaching-learning of teachers before, during and after the pandemic. The methodology was qualitative, with a narrative design. Eight mothers of families participated, from Lima, Peru in 2020, the data was collected using an interview guide. In the results, from the analysis of teacher perception and instruction, three emerging categories emerged: teacher teaching-learning before, during and after the pandemic. It was concluded that the knowledge of the mothers of families about the teaching-learning of teachers before the pandemic, was carried out in the school directly with the teacher, during the pandemic they perceive in the teachers that they have a greater commitment to improving the teachinglearning process and they understood that their support is essential in this process, after the pandemic they observe that teaching-learning must take place at school for a better interaction with their teacher, maintaining permanent communication with mothers, in order to continue supporting their children in their homes.
\end{abstract}

Keywords: long distance education; teaching; learning; pandemic; parental involvement.

International classification code: 5802.07 - Professional training.

How to cite this article:

Cucho, M., \& Carcausto, W. (2021). Mothers' narrative on emergency remote teaching-learning in initial level teachers. Revista Scientific, 6(20), 190-205, e-ISSN: 2542-2987. Recovered from: https://doi.org/10.29394/Scientific.issn.2542-2987.2021.6.20.10.190-205

Date Received: 26-12-2020
Date Acceptance: 18-03-2021
Date Publication: 05-05-2021 


\section{Introducción}

La enseñanza remota a causa de la pandemia y el aislamiento social obligatorio han reemplazado las clases presenciales, a través del uso de la tecnología y plataformas digitales. Los docentes en la actualidad se reúnen en espacios virtuales con sus estudiantes para generar aprendizajes. En este sentido, Tijo (2020): define la enseñanza remota de emergencia como una respuesta inmediata al sistema educativo sin tiempo de preparación suficiente para implementar una plataforma virtual ni preparación tecnológica para los docentes.

De acuerdo con, Hodges, Moore, Lockee, Trust y Bond (2020): muchos docentes se encuentran en un proceso de cambio que origina estrés por estar preparados para brindar una enseñanza remota, por el cual están improvisando soluciones inmediatas. Este cambio de metodología que realizan los docentes, respalda el termino de enseñanza remota de emergencia porque responde a una necesidad de aprendizaje para los estudiantes y la necesidad de crear entornos digitales de aprendizajes en un tiempo corto para los docentes. Ahora bien, Trejos-Gil y Castro-Escobar (2020): mencionan que se debe comprender el aprendizaje remoto, para responder a las necesidades y la coyuntura social que se está viviendo a causa del COVID-19, de este modo los aprendizajes deben realizarse de manera combinada, es decir trabajar para el logro de competencias, por ello se sugiere, implementar normativas precisas para mejorar el proceso de enseñanza-aprendizaje de los estudiantes.

En efecto el sistema educativo, para la Organización de las Naciones Unidas para la Educación, la Ciencia y la Cultura (UNESCO, 2020): viene implementando plataformas y recursos digitales con el fin de brindar a los estudiantes un aprendizaje de calidad. A pesar de ello, el Fondo de las Naciones Unidas para la Infancia (UNICEF, 2020): indica que existe un incremento del número de niños que no tienen acceso a la educación, 
excediendo en un $15 \%$, esto significa que, a mediados de este año, 150 millones más de niños se encuentran en esta situación problemática. Esto trae como consecuencia la interrupción del año escolar y la perdida de los aprendizajes, en los siguientes años. Aunado a ello la dificultad para el acceso de los recursos tecnológicos y el apoyo de las familias está ocasionando una brecha digital.

En este contexto se planteó como problema de investigación ¿Cuál es la percepción de madres de familias sobre la enseñanza-aprendizaje docente antes, durante y después de la pandemia?. El mismo se justificó teóricamente, porque se basa en la teoría ecológica de Bronfenbrenne (1974), citado por Sarmiento y Zapata (2014a): a nivel práctico fue valioso, porque a través de la entrevista se logra un acercamiento con las madres para conocer sus percepciones sobre la enseñanza-aprendizaje remoto de emergencia.

En ese marco, el punto de vista de Lanuza, Rizo y Saavedra (2018): refieren que los docentes y estudiantes, deben romper barreras tradicionales para adaptarse a un nuevo estilo de enseñanza-aprendizaje para lograr el desarrollo integral de la persona. Es decir que este proceso no solo se encarga de entregar y recibir información para adquirir un aprendizaje, también el uso de las TIC en la actualidad es necesario en este proceso. Con lo expuesto es notable en la actualidad, que existen muchos programas que se llevan a cabo distintas modalidades que necesita nuevos recursos para el logro de los aprendizajes.

En este contexto, las madres de familias vienen afrontando una nueva experiencia, es decir su rol juega un papel importante en el proceso de enseñanza-aprendizaje de sus hijos. Estos aprendizajes, basándose en la teoría ecológica de Bronfenbrenner (1974); y Bronfenbrenner (1979), citados por Sarmiento y Zapata (2014b): están influenciados a las prácticas predominantes en sus contextos culturales que se ajustan en la familia y la comunidad. En base a ello se toma la teoría del autor sobre la participación de 
los padres en la escuela, para desarrollar la categoría de estudio: enseñanza remoto docente, que se refiere a la construcción del aprendizaje esperado de los estudiantes, considerando el apoyo de la escuela para facilitar los recursos pedagógicos a las madres, para que puedan intervenir en el aprendizaje de sus hijos y este sea de calidad.

en este aspecto, para Sucari, Aza, Anaya y García (2019): las madres pueden influir de manera positiva o negativa en su formación, si su intervención es negativa puede traer consecuencias en el desenvolvimiento de sus hijos en las escuelas, si las madres toman el rol de supervisión, las escuelas se verán impulsadas a mejorar el proceso de aprendizaje, brindando un servicio de calidad; de lo afirmado por los autores se comprende que, al mejorar el proceso de aprendizaje, mejora la enseñanza del docente, considerando nuevos recursos contextualizados con la finalidad de propiciar la participación de la familia en el ámbito educativo. Mencionando a Miranda y Castillo (2018): la valoración que brindan los padres con respecto a la enseñanza que brinda la maestra así mismo su auto valoración con relación a ello, no debe tener en cuenta el nivel socio económico de cada familia.

Es necesario recordar a Soto-Córdova (2020): quien presenta una experiencia que realizó a través de una encuesta a los estudiantes para conocer la percepción de la enseñanza del docente, sin embargo, es necesario conocer la percepción de las madres sobre la enseñanza remota docente en el nivel inicial, para obtener mejores resultados de aprendizajes, en ese sentido podemos decir que el origen de la narrativa es la praxis de narrar acontecimientos en una línea de tiempo, para producir nuevos aprendizajes.

La narrativa en la educación percibe a los fenómenos sociales y educativos, a través de la interpretación individual que permite motivar la meditación sobre temas educativos. A esto se suma Nocetti, Hizmeri y Arriagada (2019a): que describen al relato y la narrativa como una voz crítica hacia el conocimiento, que permite expresar experiencias vividas, en este caso 
con respecto a la enseñanza remota docente.

La construcción de la realidad en la que viven las personas se refiere a las percepciones que narran según sus experiencias, basado en una lógica inter-epistemológica, esencial para la construcción de una nueva democracia, llegando a propiciar un enfoque más abierto y diverso al mundo, dentro de un sistema más justo y solidario.

Luego de una revisión bibliográfica se ha encontrado estudios como de Nocetti, Hizmeri y Arriagada (2019b): donde indica que la narrativa fomenta la praxis reflexiva y promueve la mejora en el proceso de enseñanza del docente. En esa misma línea, Viveros y Cárcamo (2019): mencionan que la relación entre la familia y escuela desde la mirada de los docentes, existen formas variadas que favorecen el incremento de espacios, resaltando la participación de tipo informativa y unidireccional, con la necesidad de mejorar su gestión institucional. Por su parte, Amar (2018): da a conocer que la investigación narrativa permite comprender un hecho, adquirir un aprendizaje entre la maestra y la familia y aprehender de sus experiencias. Parafraseando a Osorio, Mayorga, y Vargas (2019): existen dificultades en el tiempo, interés y comunicación en la relación de padres de familia y los maestros de prejardín.

A partir de lo descrito hasta el momento respecto a los estudios previos, se puede pensar que aún no existe trabajo alguno donde las madres de familias narren algo sobre el trabajo que realizan las maestras durante el estado de emergencia por el COVID-19. En virtud de ello, el presente estudio tuvo como objetivo describir los relatos de las madres de familia en cuanto a la percepción de la enseñanza-aprendizaje del docente antes y durante la pandemia.

\section{Metodología}

La investigación fue cualitativa, bajo el diseño narrativo el cual permitió explorar la experiencia de las madres de familias expresados en relatos de 
manera individual, acorde con Hernández-Sampieri y Mendoza (2018): en una cronología de tiempo (antes y durante). La población estuvo constituida por 18 madres de familia del aula de niños de cuatro años, del turno tarde de la institución educativa pública "Los libertadores" del distrito de Los Olivos, Lima, Perú. La muestra fue ocho madres de familias el cual se determinó por criterio de saturación. El tipo de muestreo fue no probabilístico por conveniencia. La recolección de datos verbales se realizó entre el mes de octubre y noviembre del año 2020.

Se empleó una guía de entrevista semiestructurada a las participantes del estudio de manera individual durante 40 minutos por el aplicativo Zoom. Previa a las entrevistas se solicitó el consentimiento de las madres. Los relatos fueron grabadas, transcritas y analizadas en una matriz de convergencia, en el cual emergieron dos subcategorías: Enseñanza-aprendizaje docente antes de la pandemia y Enseñanza-aprendizaje docente durante la pandemia.

El estudio tiene credibilidad porque durante las entrevistas, se logró recolectar datos para conocer los hallazgos, que fueron revisados por los entrevistados, para que den conformidad con su firma digital lo que ellos narraron. También es transferible porque las descripciones obtenidas fueron comparadas con los resultados de otras investigaciones. Así mismo se puede decir que tiene dependencia, porque se emplea el diseño narrativo para conocer los resultados dentro de una línea de tiempo, dando respuesta a los objetivos de estudio para obtener conclusiones.

Asimismo, la confirmabilidad de los resultados fueron analizados en una matriz de datos, registrando sus descripciones y resaltando las frases relevantes para realizar la reducción fenomenológica, luego se consideró realizar la descripción de los resultados y el análisis junto con la interpretación, permitiendo realizar las discusiones y las conclusiones. 


\section{Resultados}

A continuación, se presenta las características de las madres de familias entrevistadas, en el cual se puede observar que la mayoría tiene de 34 a 44 años, son casadas, amas de casa y estudiaron en instituciones educativas públicas, como se puede apreciar en el cuadro 1.

Cuadro 1. Características de los entrevistados.

\begin{tabular}{|l|c|}
\hline \multicolumn{1}{|c|}{ Características } & Informante clave \\
\hline Total & 8 \\
\hline Edad & 3 \\
\hline$<34$ & 1 \\
\hline 34 a 38 & 4 \\
\hline 38 a 44 & \\
\hline Estado civil & 5 \\
\hline Casado & 3 \\
\hline Soltero & \\
\hline Grado Instrucción & 4 \\
\hline Superior & 4 \\
\hline Secundaria & \\
\hline Ocupación & 4 \\
\hline Ama de casa & 2 \\
\hline Independiente & 2 \\
\hline Dependiente & 8 \\
\hline Institución educativa & \\
\hline Pública & \\
\hline
\end{tabular}

Fuente: Los Autores (2021).

\subsection{Enseñanza-aprendizaje docente antes de la pandemia}

La enseñanza del docente antes de la pandemia, hace referencia a qué y cómo enseñaba en la escuela, es decir, si las madres de familia consideran que el docente emplea estrategias pertinentes para que se produzca el aprendizaje de los estudiantes.

En tal sentido, las madres consideran que la enseñanza antes de la pandemia, era buena porque veían a sus hijos que aprendían, pero no conocían qué y cómo enseñaba la profesora, solo deducen las actividades porque algunas veces pasaban por el colegio y escuchaban lo que la profesora 
indicaba, así mismo cuando entregaban los fólderes de trabajo y por los materiales que pedían en la lista de útiles.

Solo una madre de familia, no estaba de acuerdo con la enseñanza de la profesora porque vio que su hijo no aprendió nada. Hacen referencia también que la comunicación del docente era solo por una agenda donde escribía los incidentes o solicitaba algún material, pero no les comunicaba sobre el avance de su hijo, ni lo que estaban aprendiendo en el aula. También describen que la comunicación era solo las reuniones, pero estas se realizaban con poca frecuencia. En conclusión, no había una comunicación entre padres y docentes, con respecto al aprendizaje, representado en el cuadro 2.

Cuadro 2. Entrevista a las madres sobre la enseñanza-aprendizaje del docente antes de la pandemia.

\begin{tabular}{|c|c|c|}
\hline Subcategoría & Relatos & Interpretación \\
\hline $\begin{array}{l}\text { Enseñanza-aprendizaje } \\
\text { docente antes de la } \\
\text { pandemia. }\end{array}$ & $\begin{array}{l}\text { Entrevistada 1: La verdad no } \\
\text { sabía mucho, porque toda la } \\
\text { tarea la dejaban en clase }(. . .) \\
\text { Entrevistada 2: Vi que mi hijo no } \\
\text { aprendió mucho (...) } \\
\text { Entrevistada 3: No sabía que } \\
\text { enseñaba tampoco porque no } \\
\text { nos comunicaba (...) } \\
\text { Entrevistada 4: Para mi si } \\
\text { estaba bien, porque era las } \\
\text { clases presenciales (...) } \\
\text { Entrevistada 5: Según los } \\
\text { materiales que pedía, me } \\
\text { imagino que enseñaba a pintar, } \\
\text { cortar y usar la plastilina }(. . .)\end{array}$ & $\begin{array}{l}\text { Cabe destacar, que antes } \\
\text { de la pandemia, la } \\
\text { enseñanza del docente era } \\
\text { buena, sin embargo, } \\
\text { consideran que no tenían } \\
\text { conocimiento sobre que } \\
\text { aprendían sus hijos. Esta } \\
\text { situación no permitía que } \\
\text { las familias conozcan la } \\
\text { metodología de enseñanza } \\
\text { del docente. Veían en sus } \\
\text { hijos e hijas que traían } \\
\text { novedades a casa como } \\
\text { canciones, juegos, entre } \\
\text { otros, que realizaban en el } \\
\text { colegio. }\end{array}$ \\
\hline
\end{tabular}

Fuente: Los Autores (2021).

\subsection{Enseñanza-aprendizaje docente durante de la pandemia}

La enseñanza del docente durante la pandemia, hace referencia a qué y cómo enseña de manera remota. Las madres de familias manifiestan que la enseñanza es mejor, porque la maestra está más conectada con las familias, 
está más pendiente de los niños, es muy efectiva. Sobre todo, que ellos conocen las actividades que están aprendiendo sus hijos y eso les permite reforzarlos. Una de las madres considera que es buena la enseñanza, pero la recarga de los trabajos a las madres no debería de darse, sino todo lo debería realizar la profesora directo con sus niños. Otra madre de familia considera que no es mala la enseñanza, pero debería considerar otros temas para que aprendan más los niños refiriéndose a actividades de lectura, escritura y números.

Durante la pandemia la enseñanza del docente es mucho mejor porque conocen lo que están aprendiendo sus hijos, así mismo observan el refuerzo a través de la retroalimentación, sin embargo, consideran que debería darse utilizando un medio directo entre el docente y el estudiante, para que no se genere recarga de trabajos para las madres de familias, visto en el cuadro 3.

Cuadro 3. Entrevista a las madres sobre la enseñanza-aprendizaje del docente durante la pandemia.

\begin{tabular}{|c|c|c|}
\hline Subcategoría & Relatos & Interpretación \\
\hline $\begin{array}{l}\text { Enseñanza-aprendizaje } \\
\text { docente durante la } \\
\text { pandemia. }\end{array}$ & $\begin{array}{l}\text { Entrevistada 6: Ahora la } \\
\text { enseñanza es mejor, porque el } \\
\text { mandar audios para que los } \\
\text { niños solos respondan y hablen } \\
\text { le enseñan a desenvolverse } \\
(\ldots) \\
\text { Entrevistada 7: Al inicio lo veía } \\
\text { muy básico (...) } \\
\text { Entrevistada 8: Mi hijo ha } \\
\text { aprendido mucho más que el } \\
\text { año pasado, está siendo muy } \\
\text { efectiva (...) } \\
\text { Entrevistada 9: Me parece } \\
\text { bien, pero hay mucho trabajo } \\
\text { que la mamá tienen que hacer } \\
\text { y no hay tiempo }(. . .)\end{array}$ & $\begin{array}{l}\text { Cabe resaltar que, durante la } \\
\text { pandemia, las familias tienen } \\
\text { mayor conocimiento con } \\
\text { respecto al proceso de } \\
\text { enseñanza-aprendizaje de } \\
\text { sus hijos, ya que están } \\
\text { acompañándolos en cada } \\
\text { actividad que realiza el } \\
\text { docente, también son } \\
\text { intermediarios a del } \\
\text { aprendizaje entre el docente } \\
\text { y el estudiante, porque } \\
\text { canalizan la información entre } \\
\text { ambos. Esto ha tenido } \\
\text { buenos resultados, ya que } \\
\text { valoran el trabajo del } \\
\text { docente. }\end{array}$ \\
\hline
\end{tabular}

Fuente: Los Autores (2021). 


\section{Conclusiones}

Los hallazgos resultan necesarios para conocer las experiencias de las madres en este nuevo proceso de enseñanza-aprendizaje remoto de emergencia sin precedente, con la finalidad de generar un nuevo aprendizaje a través de la reflexión narrada sobre sus vivencias en una cronología de tiempo, considerando que, si las madres conocen qué y cómo enseña el docente, mejorará la enseñanza y los resultados de aprendizaje.

La narrativa de las madres hizo que reflexionen sobre este nuevo sistema de enseñanza-aprendizaje, que permite mantener una comunicación activa, con el docente a través del WhatsApp, convirtiéndose en una herramienta digital necesaria para mejorar el apoyo en los hogares. Por ello, los hallazgos encontrados se convirtieron en el primer antecedente para futuras investigaciones, donde pueden profundizar la categoría de estudio en un contexto vulnerable o aplicarlo en otro diseño de estudio.

La percepción de las madres de familia, sobre la enseñanzaaprendizaje docente antes de la pandemia, exponen que el aprendizaje se realizaba en la escuela directamente con el docente, sin necesidad del apoyo de las familias, durante la pandemia valoran más la labor del docente, porque perciben que tienen mayor compromiso con la mejora del proceso enseñanzaaprendizaje, además comprendieron que su apoyo es fundamental en este proceso, después de la pandemia perciben que la enseñanza debe darse en la escuela para una mejor interacción con su docente, manteniendo una comunicación permanente sobre el proceso de enseñanza-aprendizaje, con la finalidad de seguir apoyando a sus hijos en sus hogares.

En la enseñanza-aprendizaje docente antes de la pandemia, las madres tenían poco conocimiento sobre lo que se enseñaba, sin embargo, consideran buena la enseñanza porque veían que sus hijos traían aprendizajes nuevos a casa, durante la pandemia consideran que la enseñanza es mucho mejor porque conocen lo que sus hijos están aprendiendo y ven el esfuerzo de la 
maestra por brindar una mejor enseñanza a distancia a pesar de las dificultades, después de la pandemia consideran que la enseñanza debe ser en la escuela con su maestra, considerando los recursos virtuales para mantener la comunicación permanente con las madres sobre el avance de sus hijos.

Entre las limitaciones de estudios se señala la falta de estudios previos sobre la categoría de estudio en el nivel inicial de la educación básica regular, porque existe una controversia sobre como la madre de familia puede evaluar a el docente, sobre su trabajo pedagógico, siendo necesarios dentro del trabajo remoto, para mejoras en el proceso de enseñanza-aprendizaje. Por ello se sugiere seguir investigando a través de otros diseños para profundizar la categoría abordada en la investigación.

\section{Referencias}

Amar, V. (2018). Déjame que mire un cuento: Narración, familia y Educación Infantil. Una investigación narrativa. Profesorado: Revista de currículum y formación del profesorado, 22(2), 389-405, eISSN: 1989-6395. Recuperado de:

https://doi.org/10.30827/profesorado.v22i2.7729

Hernández-Sampieri, R., \& Mendoza, C. (2018). Metodología de la investigación: Las rutas cuantitativa, cualitativa y mixta. ISBN: 9781-4562-6096-5. México: Editorial McGraw-Hill Education.

Hodges, C., Moore, S., Lockee, B., Trust, T., \& Bond, A. (2020). The Difference Between Emergency Remote Teaching and Online Learning. Boulder, United States: Educause Review.

Lanuza, F., Rizo, M., \& Saavedra, L. (2018). Uso y aplicación de las TIC en el proceso de enseñanza-aprendizaje. Revista Científica de FAREMEstelí, 7(25), 16-30, e-ISSN: 2305-5790. Recuperado de: https://doi.org/10.5377/farem.v0i25.5667 
Miranda, C., \& Castillo, P. (2018). Estudio metodológico de las variables que componen el constructo de "apoyo y participación de la familia en los procesos educativos”. Estudios Pedagógicos, 44(1), 115-133, e-ISSN: 0718-0705. Recuperado de: http://dx.doi.org/10.4067/S0718$\underline{07052018000100115}$

Nocetti, A., Hizmeri, J., \& Arriagada, J. (2019a,b). Narrativas, prácticas reflexivas y saberes pedagógicos en docentes en formación. Revista Panamericana de Pedagogía Saberes y Quehaceres del Pedagogo, (28), 203-228, e-ISSN: 2594-2190. Recuperado de: https://revistas.up.edu.mx/RPP/article/view/1673

Osorio, M., Mayorga, J., \& Vargas, D. (2019). Las relaciones entre padres, madres de familia y maestros en el proceso de enseñanzaaprendizaje. Bogotá, Colombia: Fundación Universitaria los Libertadores. Recuperado de: http://hdl.handle.net/11371/2259

Sarmiento, P., \& Zapata, M. (2014a,b). Modelo conceptual sobre la participación de la familia en la escuela: un estudio cualitativo en cuatro localidades del Perú. Avances de Investigación 16. Lima, Perú: Grupo de Análisis para el Desarrollo (GRADE).

Soto-Córdova, I. (2020). La relación estudiante-docente en tiempos de cuarentena: desafíos y oportunidades del aprendizaje en entornos virtuales. Revista Saberes Educativos, (5), 70-99, e-ISSN: 2452-5014. Recuperado de: https://doi.org/10.5354/2452-5014.2020.57816

Sucari, W., Aza, P., Anaya, J., \& García, J. (2019). Participación familiar en la educación escolar peruana. Revista Innova Educación, 1(1), 6-18, e-ISSN: 2664-1496. Recuperado de:

https://doi.org/10.35622/j.rie.2019.01.001

Tijo, S. (2020). Enseñanza remota de emergencia en ingeniería civil: lecciones aprendidas. ElEl: Encuentro Internacional de Educación en Ingeniería, 1-10. Colombia: Asociación Colombiana de Facultades de 
Ingeniería - ACOFI. Recuperado de:

https://acofipapers.org/index.php/eiei/article/view/781

Trejos-Gil, C. \& Castro-Escobar, H. (2020). Implicaciones ontológicas en el aprendizaje a partir del distanciamiento social impuesto por la pandemia COVID-19. Utopía y Praxis Latinoamericana, 25(extra 8), 231-247, e-ISSN: 2477-9555. Recuperado de:

https://produccioncientificaluz.org/index.php/utopia/article/view/34172 UNESCO (2020). Serie de Seminarios Web para América Latina y el Caribe: No dejar a nadie atrás en tiempos de la pandemia del COVID-19. ODS-Educación 2030. Chile: Organización de las Naciones Unidas para la Educación, la Ciencia y la Cultura.

UNICEF (2020). Evitar una generación perdida a causa de la COVID-19. ISBN: 978-92-806-5191-1. Nueva York, Estados Unidos: Fondo de las Naciones Unidas para la Infancia (UNICEF).

Viveros, L., \& Cárcamo, H. (2019). Etnografiando la relación familiaescuela. El caso de una Escuela Municipal de la Ciudad de Chillán, Chile. Revista Reflexión e Investigación Educacional, 2(1), 57-82, eISSN. 2452-4638. Recuperado de:

http://revistas.ubiobio.cl/index.php/REINED/article/view/3858 


\section{María Patricia Cucho Leyva \\ e-mail: mariacuchol@ucvvirtual.edu.pe}

Nacida en Lima, Perú, el 10 de marzo del año 1986.

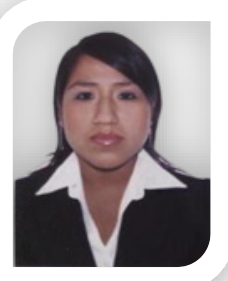

Licenciada en educación inicial, con grado de maestría en

Docencia y Gestión Educativa y doctorando en educación de la Universidad Cesar Vallejo (UCV); con experiencia a nivel universitario, nivel superior y en la Educación Básica Regular, en diversas instituciones educativas públicas y privadas del I y II del nivel inicial; reconocida a nivel de red por su destacada participación en la feria de ciencias; participé en ponencias a nivel nacional e internacional. 


\section{Wilfredo Humberto Carcausto Calla \\ e-mail: wcarcaustocalla@ucvvirtual.edu.pe}

Nací en la región de Moquegua, Perú, el 1 de septiembre

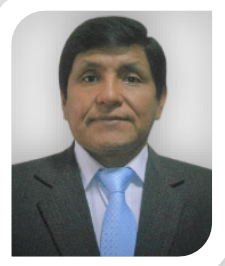
del año 1966. Obtuve el título profesional de Licenciado en Filosofía en la Universidad Nacional de San Agustín de Arequipa (UNSA); el grado Magister en problemas de aprendizaje en la Universidad Nacional de Educación "Enrique Guzmán y Valle" (UNE); y el grado de Doctor en educación en la Universidad Alas Peruanas (UAP) de la ciudad de Lima, Perú; tengo experiencia académica como docente a nivel universitario; $y$ actualmente soy investigador calificado por el Consejo Nacional de Ciencia y Tecnología e Innovación Tecnológica (CONCYTEC) del Perú.

El contenido de este manuscrito se difunde bajo una Licencia de Creative Commons ReconocimientoNoComercial-Compartirlgual 4.0 Internacional 